\title{
Antibacterial and Antifungal Activity of the Human Endometrial Fluid during the Natural Cycle
}

\author{
Marta Bregón-Villahoz $\mathbb{D}^{1},{ }^{1}$ Maria-Dolores Moragues $\mathbb{D},{ }^{1}$ Inés Arrieta-Aguirre ${ }^{\mathrm{D}},{ }^{1}$ \\ Mikel Azkargorta $\left(\mathbb{D},{ }^{2}\right.$ Lucía Lainz, ${ }^{3}$ Miren Diez-Zapirain, ${ }^{3}$ Maria Iglesias, ${ }^{3}$ \\ Maria-Begoña Prieto, ${ }^{3}$ Ana Matorras, ${ }^{4}$ Antonia Exposito, ${ }^{3}$ Felix Elortza $\mathbb{D}^{2}$, \\ and Roberto Matorras ${ }^{3,5}{ }^{3,5}$ \\ ${ }^{1}$ Department of Nursing, Faculty of Medicine and Nursing, University of the Basque Country UPV/EHU, Barrio Sarriena s/n, \\ 48940-Leioa, Spain \\ ${ }^{2}$ Proteomics Platform, CIC bioGUNE, CIBERehd, ProteoRed-ISCIII, Bizkaia Science and Technology Park, Derio, Spain \\ ${ }^{3}$ Human Reproduction Unit, Cruces University Hospital, University of the Basque Country, Barakaldo, Spain \\ ${ }^{4}$ Facultad de Medicina, Universidad Europea de Madrid, Villaviciosa de Odon, Madrid, Spain \\ ${ }^{5}$ IVIRMAI, IVI Bilbao, Spain
}

Correspondence should be addressed to Maria-Dolores Moragues; lola.moragues@ehu.eus

Received 22 September 2020; Revised 26 April 2021; Accepted 2 June 2021; Published 16 June 2021

Academic Editor: Bryan Larsen

Copyright ( 2021 Marta Bregón-Villahoz et al. This is an open access article distributed under the Creative Commons Attribution License, which permits unrestricted use, distribution, and reproduction in any medium, provided the original work is properly cited.

\begin{abstract}
Purpose. Some microbiota patterns have been associated with favorable IVF prognosis and others with pathological conditions. The endometrial fluid aspirate (EFA) contains antibacterial proteins that are enriched in implantative IVF cycles, but the antimicrobial effect of EFA has not been addressed. We aimed to evaluate the antimicrobial activity of the human endometrial fluid during the natural cycle. Methods. EFA was obtained through an embryo transfer catheter in 38 women, aged 18-40 years, with regular cycles attending to a fertility clinic. The antimicrobial activity of EFAs was tested against two strains of Staphylococcus aureus; one strain each of Streptococcus agalactiae, Enterococcus faecalis, Escherichia coli, and Klebsiella pneumoniae; and three yeasts (Candida albicans, Candida glabrata, and Candida krusei). Results. All samples exhibited antibacterial activity against S. aureus. In addition, 32.4\% of EFAs were active against one of the other microorganisms assayed, $16.2 \%$ against two, and 5.4\% against four of them. In contrast, none exhibited antibacterial activity against E. coli or K. pneumoniae. The antimicrobial activity differs considerably between EFA samples, and we failed to observe a cycle-related pattern. Conclusions. EFA presented two antimicrobial activity patterns: (a) one common to all the samples, exhibiting activity against $S$. aureus and lack of activity against E. coli and K. pneumoniae, and (b) an individualized pattern, showing activity against some of the other microorganisms tested. The intensity of antibacterial activity differs between EFA samples. Our data suggest that the uterine microbiota is controlled by means of endometrial fluid components.
\end{abstract}

\section{Introduction}

Embryo implantation is one of the most inefficient steps of assisted reproduction [1]. Implantation rates are usually $<70 \%$ even if euploid embryos are transferred [2]. Given this, there is a growing interest in investigating the uterine role in implantation. Since the early works of Noyes et al. [3], it is well known that the endometrium undergoes histological changes which are pivotal for embryo implantation.

In recent years, a huge number of different microorganisms have been reported in different organs, such as the gut, skin, lungs [4], urinary bladder [5], and vagina [6]. Some reports have focused on endometrial microbiota. It has been reported that endometrial and vaginal microbiota can differ 
in structure and composition $[7,8]$. Indeed, a microbiota pattern favorable for embryo implantation in in vitro fertilization (IVF) cycles has been described [8].

Moreover, the secretions of cultured human endometrial cells have shown antimicrobial activity [9-11], and antimicrobial peptides have been reported in them [12-14]. Nevertheless, the in vivo implication of this activity remains unknown.

We have previously shown that endometrial fluid can be easily aspirated [15] without impairing pregnancy rates, even if performed at the same time as embryo transfer [16]. Depending on the analytical approach and the applied criteria, somewhat more than 800 [17] or even over 2,200 proteins have been detected in the endometrial fluid aspirate (EFA) and some endogenous peptides of the EFA have shown antibacterial activity [18]. On the other hand, we have shown that in IVF the concentration of antibacterial peptides in the EFA is higher in cycles where implantation occurs than those where implantation fails [19].

However, actual antibacterial activity would presumably be the result of interaction between different antibacterial proteins with endogenous peptides or even with other molecules present in the endometrial fluid. Thus, we have sought to analyze the overall antibacterial activity of the EFA obtained during natural ovarian cycles.

\section{Material and Methods}

2.1. Population. The population under study consisted of 38 women of Caucasian ethnicity attending the Assisted Reproduction Unit from the Cruces University Hospital. The inclusion criteria were as follows: (i) age between 18 and 40 years; (ii) normal uterine ultrasound; (iii) cycle length of 28-30 days; (iv) previous normal cervical cytology; (v) absence of tubal conditions; (vi) no history of cervical infections, sexually transmitted diseases, pelvic inflammatory disease, endometriosis, or polycystic ovarian disease; vii) no previous miscarriages; (viii) 0-2 previous IVF cycles; (ix) no antibiotic or hormonal therapy in the last 4 months; (x) normal ovarian reserve; (xi) day of endometrial cycle 5-28; and (xii) body mass index $(\mathrm{BMI})<30 \mathrm{~kg} / \mathrm{m}^{2}$.

Patients characteristics were as follows: mean age of $35.4 \pm 3.8$ years, duration of infertility of $2.4 \pm 1.7$ years, BMI of $26.9 \pm 3.8 \mathrm{~kg} / \mathrm{m}^{2}, 94.4 \%$ having no previous children, $66.7 \%$ having no previous IVF cycles, and $22.2 \%$ smokers. In $42 \%$ of cases, the indication for IVF was the male factor.

The study was approved by our Institutional Ethical and Investigation Board (CEIC code 11/45).

2.2. Endometrial Fluid Collection. Patients were asked to participate in the study by donating an EFA sample during the mock embryo transfer, which is usually performed as a part of the pre-IVF protocol. Written informed consent was obtained.

A sterile disposable vaginal speculum was placed without employing vaginal lubricants. In cases where leucorrhea precluded access to the cervical external os, the discharge was gently removed with a sterile gauze. The EFA collection method has been recently reported $[16,19]$. An "embryo transfer" catheter (Frydman catheter, Instrumentos Médicos Estériles, SA, Spain) was inserted through the cervical canal, in aseptic conditions, without it touching the vaginal walls or the ectocervix. Abdominal ultrasound guidance was used in order to facilitate the passing through the cervix [1] and to prevent the catheter from touching the uterine fundus. The sample was aspirated with gentle negative pressure applied with a $10 \mathrm{~mL}$ syringe connected to the catheter and $50-200 \mu \mathrm{L}$ of EFA were obtained $[16,19]$. To prevent contamination with cervical fluid, aspiration was interrupted at the internal cervical os, and special care was taken to avoid traumatizing the uterine fundus or the cervix and contaminating the EFA sample with endometrial tissue or blood. Heavily blood-stained samples were discarded.

Since there is not a standardized technique to obtain EFA for antibacterial activity analysis, we used two methods. During the first part of the study, the samples ( $n=20$; EF-01 to EF-19 and EF-26) were expelled into standard cryogenic tubes, and the catheter tip was cut and placed into the same tube. In the second part of the study, after the expulsion of EFA, the inside of the catheter was rinsed with $1 \mathrm{~mL}$ of sterile saline solution. The samples were immediately frozen at $-80^{\circ} \mathrm{C}$ until processed. Prior to be processed in the laboratory, the samples of the first group were filled up to $1 \mathrm{~mL}$ final volume with sterile saline and the catheter tip was removed after vortexing for 15 seconds. The first method carried the risk of dragging some germs from the cervical canal that could produce some antimicrobial substances. In the second case, the interior washing of the catheter would avoid this potential contamination of the sample.

EFA samples were not collected by both methods in the same patient because EFA is very scarce and there was a risk that the manipulations of obtaining the first sample would affect the quality of the second one.

2.3. Assessment of Microbial Growth from EFA Samples and Identification of Isolates. Ten $\mu \mathrm{L}$ of each diluted sample, from both the "cut tip" sample group and the "flushed out catheter" group, was inoculated onto Columbia agar supplemented with $5 \%$ sheep blood (BD, USA) and incubated at $36 \pm 1{ }^{\circ} \mathrm{C}$. Another $10 \mu \mathrm{L}$ aliquot of each sample was inoculated onto Man, Rogosa, and Sharpe agar (MRS; Sigma-Aldrich, USA) and incubated at $36 \pm 1^{\circ} \mathrm{C}$ in a $5 \% \mathrm{CO}_{2}$ atmosphere.

Bacterial DNA from the most frequent colony types isolated from samples was subjected to PCR amplification of the $16 \mathrm{~S}$ rRNA gene using the bacterial universal primers $27 \mathrm{~F}$ and $1522 \mathrm{R}$ as described by Kang et al. [20]. The amplicon sequences were compared with those stored in the NCBI database to identify the isolated bacteria.

2.4. Antimicrobial Activity of EFA Samples: Broth Microdilution Assay. EFA samples were gently homogenized in a rotating wheel for $30 \mathrm{~min}$ at $4^{\circ} \mathrm{C}$ and then centrifuged at $2,500 \mathrm{~g}$ for 15 minutes to eliminate the cellular debris.

The clarified EFA samples were assayed against three Candida strains (Candida albicans SC 5314, Candida glabrata ATCC 90030, and Candida krusei ATCC 6258) and six bacterial strains (Escherichia coli CECT 434, Klebsiella pneumoniae CECT 144, Streptococcus agalactiae CECT 183T, and 
Enterococcus faecalis CECT 481 and two Staphylococcus aureus strains-the CECT 435 reference strain and a methicillin-resistant clinical isolate). ATCC stands for American Type Culture Collection (Manassas, VA, USA) and CECT for Colección Española de Cultivos Tipo (Paterna, Valencia, Spain). Candida spp. were routinely grown for maintenance in Sabouraud agar (BD) at $36 \pm 1{ }^{\circ} \mathrm{C}$ for $24 \mathrm{~h}$, while bacteria were grown in Nutrient Agar (Sigma-Aldrich) under the same conditions.

The antimicrobial activity of EFA was estimated in 96well plates, following the guidelines of the Clinical and Laboratory Standards Institute (CLSI) protocols M-27-A3 for yeasts [21], and M07-A9 for bacteria [22], adapted to our conditions. Briefly, Candida strains were inoculated in 100 $\mu \mathrm{L}$ of RPMI-MOPS, while bacteria were grown in MuellerHinton broth. Each well was supplemented with $25 \mu \mathrm{L}$ of the corresponding clarified EFA sample. Plates also included a positive growth control for each microorganism (with no EFA sample) and a negative culture medium control (no EFA sample and no microorganism), as well as growth controls for EFA samples with no added microorganisms. The plates were incubated at $36 \pm 1{ }^{\circ} \mathrm{C}$, and growth inhibition with reference to the corresponding growth positive controls was assessed visually at $24 \mathrm{~h}$ ( $48 \mathrm{~h}$ for $S$. aureus) using the following scale: -, no inhibition; +, slight inhibition (less than $50 \%$ reduction of the cell pellet in the well); ++ , moderate to high inhibition $(\geq 50 \%)$; and +++ , full growth inhibition. Growth was always blindly assessed by the same investigator (MB-V).

2.5. Statistical Analysis. The statistical association of categorical variables was analyzed with the chi-square or the Fisher's exact test, and $P$ values $<0.05$ were considered statistically significant (IBM ${ }^{\circledR}$ SPSS $^{\circledR}$ Statistics v.24).

\section{Results}

3.1. Distribution of EFA Sampling over the Menstrual Cycle. Most EFA samples (28/38) were collected in the middle of the cycle between days 13 and 19 (EF-12, sampled on day 16 , was excluded from the subsequent study of antimicrobial activity), while only five were collected between days 5 and 12 and another five in the late luteal phase, between days 24 and 28 (Table 1).

Twenty samples included the tip of the catheter, whereas the remaining 18 endometrial fluids were flushed out from the catheter. In both cases, EFA samples were made up to 1 $\mathrm{mL}$ final volume with sterile saline before being processed in the laboratory.

3.2. Microbial Growth of EFA Samples. Around 50\% of the EFA samples showed no microbial growth on Columbia agar (17/38) or MRS agar (21/38) (Table 2). The proportion of cases with microbiological growth was significantly higher for EFA samples stored with the catheter tip compared with those which were flushed out $(80 \%$ vs. $27.8 \%$ on Columbia agar $\left(\chi^{2}=8.44 ; p<0.05\right)$ and $60 \%$ vs. $27.8 \%$ on MRS agar $\left.\left(\chi^{2}=3.98 ; p<0.05\right)\right)$. The analysis of the $16 \mathrm{~S}$ rRNA gene sequences of colonies grown from 15 EFA samples showed that the most abundant and frequently isolated microbes were Gram-positive rod-shaped bacteria (Lactobacillus spp., mostly Lactobacillus gasseri), followed by colonies of Grampositive coagulase-negative staphylococci (Staphylococcus epidermidis) in five samples (EF-04, 06, 10, 12, and 26). In contrast, other colonies from EF-02, 10, and 30 that only grew on Columbia agar were identified as Actinomyces urogenitalis and Corynebacterium spp.

The frequency of positive microbial growth was 2-3 times higher for samples that had retained the catheter tip than for those that only contained the internal washing fluid of the catheter. In addition, the inoculum from three out of the five fluids with no catheter but positive growth on MRS agar only yielded 1-2 colonies. In contrast, one of the samples that included the catheter (EF-12, collected on day 16 of the menstrual cycle) yielded uncountable colonies; since excessive microorganisms interfered with the antimicrobial activity assays, this sample was discarded for further analyses.

3.3. Antimicrobial Activity of EFA Samples. The data of antimicrobial activity displayed in Table 1 are summarized in Figure 1. Almost all the EFA samples studied (34/37; $91.9 \%)$ inhibited the growth of the two S. aureus strains tested, while the remaining three samples only inhibited one of them; none of the EFA samples exhibited full growth inhibition of $S$. aureus (Table 1). In addition to inhibiting $S$. aureus, $32.4 \%(12 / 37)$ of samples were active against only one of the other microorganisms tested, $16.2 \%$ (6/37) against two microorganisms, and 5.4\% (2/37) against four microorganisms (Table 1).

Overall, $40.5 \%(15 / 37)$ of EFA samples reduced the growth of at least one of the three species of Candida, and five of them achieved full growth inhibition against them. Some other EFAs were active against S. agalactiae (13.5\%) or E. faecalis (13.5\%). In contrast, none of the diluted EFAs showed activity against E. coli or K. pneumoniae (Table 1; Figure 1).

Regarding the day of EFA collection during the menstrual cycle, all samples exhibited a certain degree of activity against $S$. aureus independently of the day of collection (Table 1). Four out of five samples that reduced the growth of E. faecalis were obtained in the central period of the cycle (days 14-17) and the fifth one on day 25 (Table 1). While $C$. glabrata and/or C. krusei were inhibited by several fluids collected at different points during the cycle (Table 1), C. albicans was inhibited only by four EFA samples from days 13 and 14; thus, the activity against $C$. albicans was statistically associated $(p<0.05)$ with EFA samples from the first part of the cycle (days 1-14) compared to the second part (days 14-28). Three EFA samples-EF04, EF06, and EF26-were active against two species of Candida (C. glabrata and C. krusei) and two-EF01 and EF27-against all three species tested (C. albicans, C. glabrata, and C. krusei).

Although care was taken to avoid epithelial bleeding during the sample collection, antibacterial activity against $S$. agalactiae was only registered in five out of 20 samples that contained traces of blood (EF-01, 05, 11, 14, and 25; Table 1).

As for the antimicrobial activity of EFA depending on the presence of culturable microorganisms in the samples, we found no differences in anti-S. aureus activity: all samples inhibited the growth of at least one of the two strains tested 
TABLE 1: Time distribution, sample characteristics and antimicrobial activity of EFA samples.

\begin{tabular}{|c|c|c|c|c|c|c|c|c|c|c|c|c|}
\hline \multirow{2}{*}{$\begin{array}{l}\text { Cycle } \\
\text { day }\end{array}$} & \multirow{2}{*}{\multicolumn{2}{|c|}{$\begin{array}{c}\text { Sample } \\
\text { Catheter } \\
\text { tip }^{b}\end{array}$}} & \multirow[b]{2}{*}{$\begin{array}{c}\text { Microbial } \\
\text { growth }\end{array}$} & \multicolumn{9}{|c|}{ Activity against microorganisms ${ }^{\mathrm{a}}$} \\
\hline & & & & $\begin{array}{l}\text { E. } \\
\text { coli }\end{array}$ & $\begin{array}{c}\text { K. } \\
\text { pneumoniae }\end{array}$ & $\begin{array}{c}\text { E. } \\
\text { faecalis }\end{array}$ & $\begin{array}{c}S . \\
\text { agalactiae }\end{array}$ & $\begin{array}{c}S . \\
\text { aureus }\end{array}$ & MRSA & $\begin{array}{c}C . \\
\text { albicans }\end{array}$ & $\begin{array}{c}\text { C. } \\
\text { glabrata }\end{array}$ & $\begin{array}{c}\text { C. } \\
\text { krusei }\end{array}$ \\
\hline 5 & EF-05 & Yes & Yes & - & - & - & + & - & + & - & - & + \\
\hline \multirow{2}{*}{10} & EF-07 & Yes & Yes & - & - & - & - & ++ & ++ & - & - & - \\
\hline & EF-22 & No & No & - & - & - & - & + & + & - & - & - \\
\hline 11 & EF-24 & No & Yes & - & - & - & - & + & - & - & - & +++ \\
\hline 12 & EF-23 & No & No & - & - & - & - & + & ++ & - & - & - \\
\hline \multirow{5}{*}{13} & EF-01 & Yes & Yes & - & - & - & ++ & ++ & ++ & +++ & +++ & +++ \\
\hline & EF-02 & Yes & Yes & - & - & - & - & + & ++ & - & - & - \\
\hline & EF-10 & Yes & Yes & - & - & - & - & ++ & + & - & ++ & - \\
\hline & EF-28 & No & No & - & - & - & - & - & + & ++ & - & - \\
\hline & EF-29 & No & No & - & - & - & - & ++ & + & - & - & - \\
\hline \multirow{5}{*}{14} & EF-15 & Yes & Yes & - & - & - & - & + & + & - & - & - \\
\hline & EF-20 & No & No & - & - & - & - & ++ & + & - & - & - \\
\hline & EF-21 & No & Yes & - & - & - & - & + & ++ & +++ & - & - \\
\hline & EF-25 & No & No & - & - & - & + & ++ & ++ & - & - & - \\
\hline & EF-27 & No & No & - & - & + & - & + & ++ & +++ & +++ & +++ \\
\hline \multirow{4}{*}{15} & EF-04 & Yes & Yes & - & - & - & - & + & ++ & - & ++ & + \\
\hline & EF-19 & Yes & Yes & - & - & - & - & + & + & - & ++ & - \\
\hline & EF-30 & No & Yes & - & - & - & - & ++ & ++ & - & - & - \\
\hline & EF-37 & No & No & - & - & - & - & ++ & + & - & - & - \\
\hline \multirow{7}{*}{16} & EF-03 & Yes & Yes & - & - & - & - & + & ++ & - & - & + \\
\hline & EF-13 & Yes & Yes & - & - & + & - & + & + & - & - & - \\
\hline & EF-16 & Yes & No & - & - & + & - & ++ & ++ & - & - & - \\
\hline & EF-26 & Yes & Yes & - & - & - & - & + & ++ & - & + & ++ \\
\hline & EF-31 & No & Yes & - & - & - & - & ++ & + & - & - & - \\
\hline & EF-35 & No & Yes & - & - & - & - & ++ & ++ & - & - & - \\
\hline & EF-36 & No & Yes & - & - & - & - & ++ & ++ & - & - & - \\
\hline \multirow{2}{*}{17} & EF-33 & No & No & - & - & + & - & ++ & + & - & - & - \\
\hline & EF-34 & No & No & - & - & - & - & + & + & - & - & - \\
\hline \multirow{2}{*}{18} & EF-06 & Yes & Yes & - & - & - & - & + & + & - & +++ & + \\
\hline & EF-14 & Yes & Yes & - & - & - & +++ & + & + & - & - & - \\
\hline \multirow{2}{*}{19} & EF-18 & Yes & Yes & - & - & - & - & + & + & - & - & - \\
\hline & EF-32 & No & No & - & - & - & - & + & + & - & - & - \\
\hline 24 & EF-17 & Yes & No & - & - & - & - & + & + & - & - & - \\
\hline 25 & EF-11 & Yes & Yes & - & - & - & + & ++ & ++ & - & ++ & - \\
\hline 26 & EF-08 & Yes & Yes & - & - & - & - & + & ++ & - & - & + \\
\hline 27 & EF-09 & Yes & Yes & - & - & + & - & ++ & + & - & ++ & - \\
\hline 28 & EF-38 & No & No & - & - & - & - & ++ & + & - & - & - \\
\hline
\end{tabular}

${ }^{a}$ Antimicrobial activity with reference to the cell pellet of the positive growth control well. -: no inhibition; +: slight inhibition (<50\% reduction); ++: moderate to high inhibition ( $\geq 50 \%) ;+++$ : full growth inhibition. ${ }^{b}$ Catheter: "yes" stands for samples stored with the catheter tip prior to processing; "no" stands for samples flushed out from the catheter before being stored. Microorganisms: Escherichia coli, Klebsiella pneumoniae, Enterococcus faecalis, Streptococcus agalactiae, Staphylococcus aureus, MRSA (Methicillin-resistant S. aureus), Candida albicans, Candida glabrata, and Candida krusei.

(Table 1). However, antimicrobial activity against the rest of microorganisms tested was somewhat more frequent in cases where the microbial culture of EFA on MRS and/or Colum- bia agar was positive $(15 / 23 ; 65.22 \%)$ than those with no culturable bacteria $(5 / 14 ; 35.71 \%) \quad\left(\chi^{2}=3.05 ; p=0.08\right)$ (Table 1$)$. In regard to the collection method and storage, 
TABLE 2: Microbial growth of endometrial fluid aspirate (EFA) samples from 38 women on Columbia agar and MRS agar. The ratio of EFA samples displaying microbial growth in both media was significantly higher for those stored with the catheter tip $\left({ }^{*} \chi^{2}=8.44, p<0.05\right.$; ${ }^{* *}$ $\left.\chi^{2}=3.98, p<0.05\right)$ compared to EFA samples flushed out from the catheter.

\begin{tabular}{|c|c|c|c|c|c|c|c|c|c|}
\hline \multirow[b]{4}{*}{ EFA samples } & \multirow[b]{4}{*}{$N$} & \multicolumn{8}{|c|}{ Microbial growth of EFA samples on } \\
\hline & & \multicolumn{4}{|c|}{ Columbia agar } & \multicolumn{4}{|c|}{ MRS agar } \\
\hline & & \multicolumn{2}{|c|}{ Positive } & \multicolumn{2}{|c|}{ Negative } & \multicolumn{2}{|c|}{ Positive } & \multicolumn{2}{|c|}{ Negative } \\
\hline & & $N$ & $\%$ & $N$ & $\%$ & $N$ & $\%$ & $N$ & $\%$ \\
\hline With catheter tip & 20 & 16 & $80^{*}$ & 4 & 20 & 12 & $60^{* *}$ & 8 & 40 \\
\hline Flushed out from catheter & 18 & 5 & 27.8 & 13 & 72.2 & 5 & 27.8 & 13 & 72.2 \\
\hline Total & 38 & 21 & 55.3 & 17 & 44.7 & 17 & 44.7 & 21 & 55.3 \\
\hline
\end{tabular}

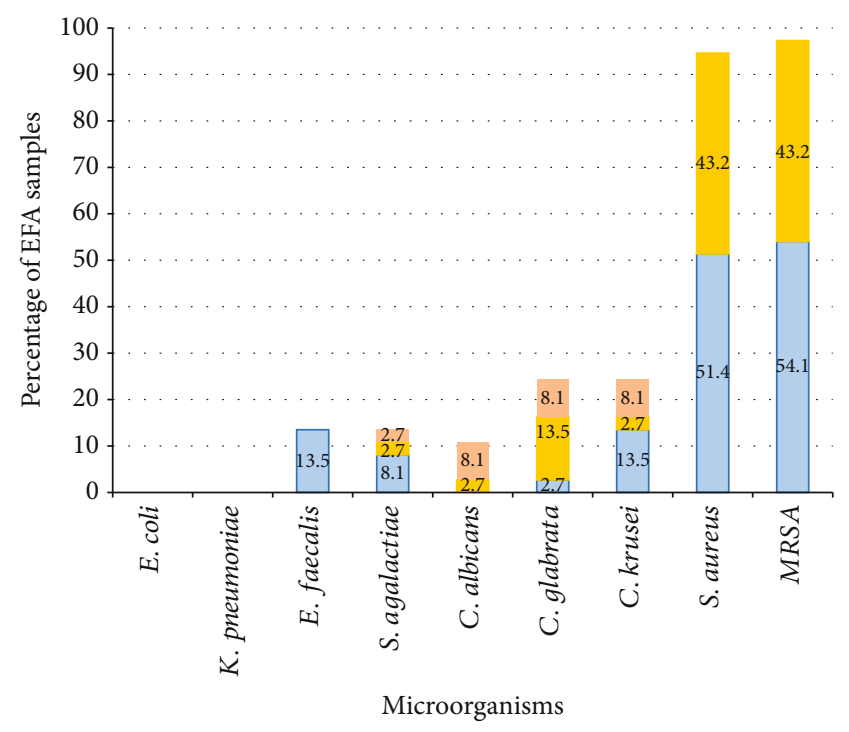

FIgURE 1: Percentage of EFA samples with antimicrobial activity against different microorganisms. Color code refers to the intensity of inhibition as described in Material and Methods (blue: +; yellow: ++; salmon: +++).

samples that included the catheter tip were more frequently associated with activity against microorganisms other than S. aureus $(14 / 19 ; 73.68 \%)$ than those that contained only the flushing fluid from inside the catheter $(6 / 18 ; 33.33 \%)$, and this association was statistically significant $\left(\chi^{2}=6.06\right.$; $p<0.05)$.

\section{Discussion}

Microbial populations of varied composition have been described in association with different anatomical locations of the human body [23]. The interaction between the host and microbiota has been shown to play an essential role in many aspects of human physiology [24-26].

Much less is known about the uterine side of implantation than the embryonic side. The challenges of investigating the uterine side of implantation include endometrial cyclic changes, intercycle variability, and the influence of ovarian stimulation on endometrial changes, as well as the potential adverse effect of endometrial biopsy on implantation, if performed close to the time of embryo transfer [16]. In this context, we have focused our study on EFA, which can be obtained at the same time as embryo transfer, without impairing implantation rates [16].

Moreno et al. [8] identified a favorable pattern in the endometrial microbiota for the successful implantation of the embryo in IVF, characterized by a high proportion of Lactobacillus. In a recent study focusing on the protein composition of EFA obtained at the same time as the embryo transfer, we showed that EFA from IVF cycles where implantation took place ("implantative" cycles) was richer in antibacterial proteins than EFA from "nonimplantative" cycles [19]. This is in agreement with very recent data from an endogenous peptidomics-focused mass spectrometry analysis of EFA describing the presence of a number of peptides with potential antibacterial activity in this fluid [18]. In line with this, selected in silico predicted antibacterial peptides were synthesized and tested in vitro for antimicrobial activity. Preliminary results showed that, indeed, some of these peptides present antibacterial activity [18]. In the same way, it has recently been suggested that the innate immune system senses pathogen-associated molecular patterns and this could induce the release of antimicrobial peptides into the uterine cavity [27].

In the present study, we have evaluated the antimicrobial activity of EFA, which contains many different antibacterial proteins and peptides, whose synergisms or even antagonisms are still unknown. Indeed, some microorganisms present in EFA could also play an antibacterial role. For instance, the Lactobacillus genus produces lactic acid and short-chain fatty acids, acidifying the environment to $\mathrm{pH} \leq 4.5$ in the vagina and prohibiting the growth of other pathogenic or dysbiotic bacteria in healthy women [28, 29]. However, concerning the endometrium, no correlation has been observed between the $\mathrm{pH}$ value and the endometrial microbiota [8].

The possibility of sample contamination is a significant hurdle to investigate uterine microbiota [30]. There is no standardized methodology for the study of human uterine microbiota [30]. In most of the studies, the samples were collected through the cervix [30]. Some authors used endometrial biopsies [31], others used transcervical aspiration through an embryo transfer catheter $[8,32-34]$ and then emptied the content (without flushing out the catheter), others used a double lumen catheter, without aspiration, and analyzed the distal portion of the transfer catheter [7, $35]$, and some combined a double lumen ET catheter with lavage and endometrial biopsy [36]. Aspiration of EF under aseptic conditions has been shown to be a safe and effective 
method to evaluate the endometrial microbiota $[8,37]$. We have undertaken this work in order to evaluate the potential antimicrobial activity of the endometrial fluid of 38 women in reproductive age. The EFA samples were collected through aspiration with an embryo transfer catheter passed through the cervical os, taking precautions to avoid contamination with the cervix microbiota. Two different collection methods were used: (i) emptying the catheter content and storing the sample with the catheter tip and (ii) flushing the inside of the catheter with saline solution. In our work, approximately half of the EFA samples contained culturable microorganisms, in agreement with data stating that the endometrial cavity is not sterile in most healthy women [38].

Twice as many positive cultures were obtained from samples stored with the catheter tip compared to samples without it. Colonies of Staphylococcus epidermidis were only identified in those samples stored with the catheter. It could be speculated that the external surface of the catheter retained a number of microorganisms from the cervical canal, and hence, for microbiological analyses, it seems advisable to collect only the internal fluid of the catheter. Nevertheless, the viable bacteria identified in both sample types consisted mainly of Lactobacillus spp. and to a much lesser extent Actinomyces urogenitalis and Corynebacterium spp. Lactobacillus spp. were the most frequently isolated bacteria from the EFA samples, in agreement with previous reports of Wee et al. [39] and Moreno and Simon [37]. Although significant differences have been reported in vaginal and uterine microbiota $[8,33,34,40]$, as far as we know, cervical and uterine microbiota have not been compared. Selman et al. studied endocervical and ectocervical samples as well as the internal tip of the catheter, but they did not aspirate samples and the results for each localization were not compared [35].

There is also a controversy about the method of analysis of the uterine microbiome. Regarding the culture of viable microbes, less than $1 \%$ of the bacteria present in a sample grow and form colonies; furthermore, some human samples may contain a limited amount of microorganisms [41]. On the other hand, the next-generation sequencing (NGS) analysis of EFA samples containing a small number of microorganisms may be distorted by abundant contaminating vaginal microbiota [30]. Moreover, NGS readings do not differentiate between live and dead microorganisms, and consequently, the uterine microbial population may be overestimated.

The uterine microbiota have been reported to fluctuate with menstrual cycle timing [42], ovarian stimulation [43], ethnic group [44], certain pathological conditions [42], and IVF prognosis [37]. In our study, performed in natural cycles of Caucasian women in reproductive age, we have not been able to establish a clear association between the viable microbiota of EFA samples and the time of sampling during the menstrual cycle. However, we acknowledge that only a small number of the samples studied were outside the central part of the cycle.

Regarding the antimicrobial activity, all the EFA samples in the present work inhibited the in vitro growth of $S$. aureus to some extent, and there were no significant differences between culturable and nonculturable samples, or even between samples collected at different times over the menstrual cycle. A number of potential antibacterial peptides have been reported from human endometrial tissue cultures [12-14]. Our results are consistent with those obtained with cultures of uterine epithelial cells [45], showing the production of antibacterial factors that effectively killed S. aureus, and correlation with secretory leukocyte protease inhibitor concentrations. Nevertheless, in the same study on uterine epithelial cell culture [45], antibacterial activity against E. coli was also observed, a type of activity that was not observed in any patients from our study. We were also unable to find any activity against $K$. pneumoniae in any of our patients' EFA samples. On the other hand, more than half of the EFA samples of the studied women (54.1\%) exhibited antimicrobial activity against other microorganisms including C. albicans, C. glabrata, C. krusei, S. agalactiae, and/or E. faecalis. Moreover, our findings regarding C. albicans are in agreement with those of Wira et al. [46], who reported that secretions of cultures from the upper female reproductive tract cells inhibit yeast and hyphal forms of C. albicans.

It has to be stressed that there was a remarkable heterogeneity in antimicrobial activity patterns: (i) the intensity of the antimicrobial activity differs notably between the different specimens, and (ii) apart from S. aureus which was always inhibited, the proportion of EFA inhibiting some of the remaining microorganisms assayed ranged from 10.8 to $24.3 \%$. Furthermore, in addition to S. aureus, $32.4 \%$ of EFA samples were active against only one of the remaining microorganisms assayed, $16.2 \%$ against two microorganisms, and only $5.4 \%$ against four microorganisms. On the other hand, it must be highlighted that a number of microorganisms, especially anaerobic bacteria, were not evaluated in our study.

Concerning the source of antibacterial compounds, in addition to endometrial tissue, as suggested by tissue culture studies, one cannot rule out an associated effect coming from the microbiota of the endometrial cavity. In agreement with this, apart from the anti-S. aureus activity which was present in every sample, we found that antimicrobial activity against the other tested microorganisms was more frequent in the samples where microbiological cultures were positive. Wang et al. [47] attributed antimicrobial activity to Lactobacillus spp. that produces lactic acid and hydrogen peroxide, among other compounds, which can inhibit the growth of potentially pathogenic bacteria, as well as the filamentation of $C$. albicans. Nonetheless, the anaerobic environment of the endometrium does not facilitate hydrogen peroxide production by Lactobacillus. Indeed, we have documented anti-Candida activity in more than one-third of the EFA samples, even in cases when no growth of Lactobacillus on MRS medium was recorded. Regarding the method of collection and storage, the samples containing only the fluid flushed out from the catheter were associated with the least frequently positive cultures and antimicrobial activity. Although our sample numbers were limited and we were unable to assess a range of confounding factors, the latter method is probably the one that can provide the most reliable information on the uterine microbiota and its antimicrobial activity. 
In this study, we have shown that EFA samples from women of reproductive age exhibit antimicrobial activity against some microorganisms that could impair IVF results. All the EFA samples tested in vitro reduced the growth of S. aureus, and many of them inhibited Candida spp., E. faecalis, and/or S. agalactiae as well, but not E. coli or K. pneumoniae. To the best of our knowledge, this is the first report on the direct antimicrobial activity of endometrial fluid aspirate samples in humans.

Further studies are warranted to characterize the endometrial fluid of women undergoing IVF procedures in order to assess whether there is a relationship between the antimicrobial activity of raw EFA and implantation success.

\section{Data Availability}

The data used to support the findings of this study are available from the corresponding author upon request.

\section{Ethical Approval}

The study was approved by our Institutional Ethical and Investigation Board (CEIC code 11/45).

\section{Consent}

Patients were asked to participate in the study by donating an EFA sample during the mock embryo transfer, which is usually performed as a part of the pre-IVF protocol. Written informed consent was obtained.

\section{Conflicts of Interest}

The authors report no conflict of interest.

\section{Authors' Contributions}

M Bregón-Villahoz is responsible for the sample analyses, data collection, and manuscript writing. MD Moragues is assigned to the protocol development, data analysis, and manuscript writing/editing. I Arrieta-Aguirre is responsible for the samples and data analysis. L Lainz, M Diez-Zapirain, M Iglesias, A Exposito, and MB Prieto are assigned to patient selection and sample collection. A Matorras, F Elortza, and M Azkargorta did the data analysis. R Matorras is responsible for the project development, patient selection, sample collection, data management and analysis, and manuscript writing/editing.

\section{Acknowledgments}

This study was partially supported by a Grant for Fertility Innovation (GFI, 2011) from Merck, Darmstadt, Germany. M. Bregón-Villahoz is recipient of a predoctoral grant from the Universidad del País Vasco-Euskal Herriko Unibertsitatea (UPV/EHU) (PIF19/316). The authors thank the technical and human support provided by DNA Bank Service (SGIker) of the University of the Basque Country (UPV/EHU) and European funding (ERDF and ESF). CIC bioGUNE is accredited with the Severo Ochoa Excellence award by the Spanish Ministerio de Economía y Competitividad, MINECO (SEV-2016-0644).

\section{References}

[1] R. Matorras, E. Urquijo, R. Mendoza, B. Corcostegui, A. Exposito, and F. J. Rodriguez-Escudero, "Ultrasoundguided embryo transfer improves pregnancy rates and increases the frequency of easy transfers," Human Reproduction, vol. 17, no. 7, pp. 1762-1766, 2002.

[2] J. Friedenthal, S. M. Maxwell, S. Munné et al., "Next generation sequencing for preimplantation genetic screening improves pregnancy outcomes compared with array comparative genomic hybridization in single thawed euploid embryo transfer cycles," Fertility and Sterility, vol. 109, no. 4, pp. 627-632, 2018.

[3] R. W. Noyes, A. T. Hertig, and J. Rock, "Dating the endometrial biopsy," Obstetrical \& Gynecological Survey, vol. 5, no. 4, pp. 561-564, 1950.

[4] R. P. Dickson, J. R. Erb-Downward, and G. B. Huffnagle, "Homeostasis and its disruption in the lung microbiome," American Journal of Physiology. Lung Cellular and Molecular Physiology, vol. 309, no. 10, pp. L1047-L1055, 2015.

[5] L. Brubaker and A. J. Wolfe, "The new world of the urinary microbiota in women," American Journal of Obstetrics and Gynecology, vol. 213, no. 5, pp. 644-649, 2015.

[6] W. Mendling, "Vaginal microbiota," Advances in Experimental Medicine and Biology, vol. 902, pp. 83-93, 2016.

[7] J. M. Franasiak, M. D. Werner, C. R. Juneau et al., "Endometrial microbiome at the time of embryo transfer: nextgeneration sequencing of the $16 \mathrm{~S}$ ribosomal subunit," Journal of Assisted Reproduction and Genetics, vol. 33, no. 1, pp. 129136, 2016.

[8] I. Moreno, F. M. Codoñer, F. Vilella et al., "Evidence that the endometrial microbiota has an effect on implantation success or failure," American Journal of Obstetrics and Gynecology, vol. 215, no. 6, pp. 684-703, 2016.

[9] J. V. Fahey, T. M. Schaefer, J. Y. Channon, and C. R. Wira, "Secretion of cytokines and chemokines by polarized human epithelial cells from the female reproductive tract," Human Reproduction, vol. 20, no. 6, pp. 1439-1446, 2005.

[10] D. O. Ochiel, J. V. Fahey, M. Ghosh, S. N. Haddad, and C. R. Wira, "Innate immunity in the female reproductive tract: role of sex hormones in regulating uterine epithelial cell protection against pathogens," Curr Womens Health Rev, vol. 4, no. 2, pp. $102-117,2008$.

[11] A. W. Horne, S. J. Stock, and A. E. King, "Innate immunity and disorders of the female reproductive tract," Reproduction, vol. 135, no. 6, pp. 739-749, 2008.

[12] Y. Feng, X. Pan, N. Huang, Y. Feng, Q. Wu, and B. Wang, "The human beta-defensins expression in female genital tract and pregnancy-related tissues," Sichuan Da Xue Xue Bao. Yi Xue Ban, vol. 34, no. 2, pp. 217-219, 2003.

[13] A. E. King, H. O. Critchley, and R. W. Kelly, "Innate immune defences in the human endometrium," Reproductive Biology and Endocrinology, vol. 1, no. 1, pp. 116-116, 2003.

[14] V. L. Yarbrough, S. Winkle, and M. M. Herbst-Kralovetz, "Antimicrobial peptides in the female reproductive tract: a critical component of the mucosal immune barrier with physiological and clinical implications," Human Reproduction Update, vol. 21, no. 3, pp. 353-377, 2015. 
[15] A. Ametzazurra, R. Matorras, J. A. Garcia-Velasco et al., "Endometrial fluid is a specific and non-invasive biological sample for protein biomarker identification in endometriosis," Human Reproduction, vol. 24, no. 4, pp. 954-965, 2009.

[16] R. Matorras, S. Quevedo, B. Corral et al., "Proteomic pattern of implantative human endometrial fluid in in vitro fertilization cycles," Archives of Gynecology and Obstetrics, vol. 297, no. 6, pp. 1577-1586, 2018.

[17] J. Casado-Vela, E. Rodriguez-Suarez, I. Iloro et al., "Comprehensive proteomic analysis of human endometrial fluid aspirate," Journal of Proteome Research, vol. 8, no. 10, pp. 46224632, 2009.

[18] M. Azkargorta, M. Bregón-Villahoz, I. Escobes et al., "In-depth proteomics and natural peptidomics analyses reveal antibacterial peptides in human endometrial fluid," Journal of Proteomics, vol. 216, article 103652, 2020.

[19] M. Azkargorta, I. Escobes, I. Iloro et al., "Differential proteomic analysis of endometrial fluid suggests increased inflammation and impaired glucose metabolism in non-implantative IVF cycles and pinpoints PYGB as a putative implantation marker," Human Reproduction, vol. 33, no. 10, pp. 18981906, 2018.

[20] M. Kang, H. Lim, J. Oh et al., "Antimicrobial activity of Lactobacillus salivarius and Lactobacillus fermentum against Staphylococcus aureus," Pathogens and disease, vol. 75, no. 2, 2017.

[21] Clinical and Laboratory Standards Institute, (CLSI), M27-A3. Reference Method for Broth Dilution Antifungal Susceptibility Testing of Yeasts, Clinical and Laboratory Standards Institute, Wayne, PA, 2008.

[22] Clinical and Laboratory Standards Institute, (CLSI) (2012) M07-A9, "Methods for Dilution Antimicrobial Susceptibility Tests for Bacteria That Grow Aerobically," in Approved StandardClinical and Laboratory Standards Institute.

[23] D. Ribet and P. Cossart, "How bacterial pathogens colonize their hosts and invade deeper tissues," Microbes and infection, vol. 17, no. 3, pp. 173-183, 2015.

[24] L. Dethlefsen, M. McFall-Ngai, and D. A. Relman, “An ecological and evolutionary perspective on human-microbe mutualism and disease," Nature, vol. 449, no. 7164, pp. 811-818, 2007.

[25] I. Cho and M. J. Blaser, "The human microbiome: at the interface of health and disease," Nature Reviews. Genetics, vol. 13, no. 4, pp. 260-270, 2012.

[26] H. Verstraelen, R. Vilchez-Vargas, F. Desimpel et al., "Characterisation of the human uterine microbiome in non-pregnant women through deep sequencing of the V1-2 region of the 16S rRNA gene," PeerJ, vol. 4, article e1602, 2016.

[27] M. Benner, G. Ferwerda, I. Joosten, and R. G. van der Molen, "How uterine microbiota might be responsible for a receptive, fertile endometrium," Human Reproduction Update, vol. 24, no. 4, pp. 393-415, 2018.

[28] A. Skarin and J. Sylwan, "Vaginal lactobacilli inhibiting growth of Gardnerella vaginalis, Mobiluncus and other bacterial species cultured from vaginal content of women with bacterial vaginosis," Acta Pathologica, Microbiologica, et Immunologica Scandinavica. Section B, vol. 94B, no. 1-6, pp. 399-403, 1986.

[29] T. Yamamoto, X. Zhou, C. J. Williams, A. Hochwalt, and L. J. Forney, "Bacterial populations in the vaginas of healthy adolescent women," Journal of Pediatric and Adolescent Gynecology, vol. 22, no. 1, pp. 11-18, 2009.
[30] J. M. Baker, D. M. Chase, and M. M. Herbst-Kralovetz, "Uterine microbiota: residents, tourists, or invaders?," Frontiers in Immunology, vol. 9, p. 208, 2018.

[31] K. Kitaya, Y. Nagai, W. Arai, Y. Sakuraba, and T. Ishikawa, "Characterization of microbiota in endometrial fluid and vaginal secretions in infertile women with repeated implantation failure," Mediators of inflammation, vol. 2019, 10 pages, 2019.

[32] F. Vilella, L. Ramirez, O. Berlanga et al., "PGE2 and PGF2 $\alpha$ concentrations in human endometrial fluid as biomarkers for embryonic implantation," The Journal of Clinical Endocrinology and Metabolism, vol. 98, no. 10, pp. 4123-4132, 2013.

[33] K. Kyono, T. Hashimoto, Y. Nagai, and Y. Sakuraba, “Analysis of endometrial microbiota by $16 \mathrm{~S}$ ribosomal RNA gene sequencing among infertile patients: a single-center pilot study," Reprod Med Biol, vol. 17, no. 3, pp. 297-306, 2018.

[34] K. Kyono, T. Hashimoto, S. Kikuchi, Y. Nagai, and Y. Sakuraba, "A pilot study and case reports on endometrial microbiota and pregnancy outcome: an analysis using 16S rRNA gene sequencing among IVF patients, and trial therapeutic intervention for dysbiotic endometrium," Reprod Med Biol, vol. 18, no. 1, pp. 72-82, 2019.

[35] H. Selman, M. Mariani, N. Barnocchi et al., "Examination of bacterial contamination at the time of embryo transfer, and its impact on the IVF/pregnancy outcome," Journal of Assisted Reproduction and Genetics, vol. 24, no. 9, pp. 395-399, 2007.

[36] Y. Liu, K. K. W. Wong, E. Y. L. Ko et al., "Systematic comparison of bacterial colonization of endometrial tissue and fluid samples in recurrent miscarriage patients: implications for future endometrial microbiome studies," Clinical Chemistry, vol. 64, no. 12, pp. 1743-1752, 2018.

[37] I. Moreno and C. Simon, "Relevance of assessing the uterine microbiota in infertility," Fertility and Sterility, vol. 110, no. 3, pp. 337-343, 2018.

[38] C. M. Mitchell, A. Haick, E. Nkwopara et al., "Colonization of the upper genital tract by vaginal bacterial species in nonpregnant women," American journal of obstetrics and gynecology, vol. 212, no. 5, pp. 611.e1-611.e9, 2015.

[39] B. A. Wee, M. Thomas, E. L. Sweeney et al., "A retrospective pilot study to determine whether the reproductive tract microbiota differs between women with a history of infertility and fertile women," The Australian \& New Zealand Journal of Obstetrics \& Gynaecology, vol. 58, no. 3, pp. 341-348, 2018.

[40] T. Haahr, P. Humaidan, H. O. Elbaek et al., "Vaginal microbiota and in vitro fertilization outcomes: development of a simple diagnostic tool to predict patients at risk of a poor reproductive outcome," The Journal of Infectious Diseases, vol. 219, no. 11, pp. 1809-1817, 2019.

[41] X. Tao, J. M. Franasiak, Y. Zhan et al., "Characterizing the endometrial microbiome by analyzing the ultra-low bacteria from embryo transfer catheter tips in IVF cycles: next generation sequencing (NGS) analysis of the $16 \mathrm{~S}$ ribosomal gene," Human Microbiome Journal, vol. 3, pp. 15-21, 2017.

[42] C. Chen, X. Song, W. Wei et al., "The microbiota continuum along the female reproductive tract and its relation to uterine-related diseases," Nature Communications, vol. 8, no. 1, p. 875, 2017.

[43] K. N. Khan, A. Fujishita, H. Masumoto et al., "Molecular detection of intrauterine microbial colonization in women with endometriosis," European Journal of Obstetrics, Gynecology, and Reproductive Biology, vol. 199, pp. 69-75, 2016. 
[44] J. Ravel, P. Gajer, Z. Abdo et al., "Vaginal microbiome of reproductive-age women," Proceedings of the National Academy of Sciences of the United States of America, vol. 108, Supplement_1, pp. 4680-4687, 2011.

[45] J. V. Fahey and C. R. Wira, "Effect of menstrual status on antibacterial activity and secretory leukocyte protease inhibitor production by human uterine epithelial cells in culture," The Journal of Infectious Diseases, vol. 185, no. 11, pp. 16061613, 2002.

[46] C. R. Wira, M. V. Patel, M. Ghosh, L. Mukura, and J. V. Fahey, "Innate immunity in the human female reproductive tract: endocrine regulation of endogenous antimicrobial protection against HIV and other sexually transmitted infections," American Journal of Reproductive Immunology, vol. 65, no. 3, pp. 196-211, 2011.

[47] S. Wang, Q. Wang, E. Yang, L. Yan, T. Li, and H. Zhuang, "Antimicrobial compounds produced by vaginal Lactobacillus crispatus are able to strongly inhibit Candida albicans growth, hyphal formation and regulate virulence-related gene expressions," Frontiers in Microbiology, vol. 8, p. 564, 2017. 